\title{
OIFITS version 2: the new standard for optical/IR interferometry data exchange
}

\author{
Gilles Duvert ${ }^{\mathrm{a}}$, John S. Young ${ }^{\mathrm{b}}$, and Christian Hummel ${ }^{\mathrm{c}}$ \\ ${ }^{a}$ Univ. Grenoble Alpes, IPAG, F-38000 Grenoble, France and CNRS, IPAG, F-38000 \\ Grenoble, France \\ ${ }^{\mathrm{b}}$ Cavendish Laboratory, University of Cambridge, J J Thompson Avenue, Cambridge, UK. \\ ${ }^{c}$ European Southern Observatory, Karl-Schwarzschild-Str. 2, 85748 Garching
}

\begin{abstract}
We present the second version of the OI Exchange Format (OIFITS2), the standard for exchanging calibrated data from optical (visible/infrared) interferometers. This new version provides definitions of several new tables addressing the needs of future interferometric instruments such as GRAVITY and MATISSE, optional data columns for a more rigorous description of measurement errors and their correlations and various new header keywords for book-keeping and data discovery. In this paper, we outline the new features of OIFITS2 and describe their anticipated usage. Some considerations not present in the normative text are also discussed.
\end{abstract}

Keywords: normalization, data format, optical interferometry, virtual observatory

\section{INTRODUCTION}

The common data format for exchange of interferometric measurements in optical and infrared interferometry (commonly known as OIFITS) was published in $2005^{?}$. The format specification was the result of a five years maturation process, starting in 2000 under the auspices of the International Astronomical Union with the contribution of many individuals and the then existing optical interferometer projects (COAST, NPOI, IOTA, CHARA, VLTI, PTI, KI).

It is an understatement to say that the existence of OIFITS, and the observance of this norm by data producers, has been pivotal not only in the dissemination of optical interferometry data, but also in the facilitation of additional contributions by the community, namely observation preparation tools, data reduction, data interpretation and data mining tools. The fact that software for reading, writing and merging OIFITS files was freely available at the time of publication certainly helped to facilitate the adoption of the standard. It was especially useful because OIFITS, as with any data container, is not intended to be especially readable by humans. At the time of adoption of OIFITS, the Virtual Observatory (VO) efforts towards standardisation and interoperability were still in their infancy, and FITS was a natural and traditional choice, also due to the availability of reader and writer programs in various scientific languages.

Eventually the scope of the format became too narrow with respect to its usage "in the wild," and a revision of the standard became necessary. A new version, OIFITS v.2 has recently been released? . In the following sections we examine the intent behind the changes in the format.

\section{OPTICAL INTERFEROMETRY OBSERVABLES}

In astronomy, interferometers measures complex coherent fluxes $C_{(u, v)}$ between pairs of telescopes ("apertures"), whose distance is a vector of coordinates $(u, v)$. These are related to the Fourier Transform of the brightness distribution of the (small) patch of sky around the object $O(x, y)$ observed by all telescopes simultaneously, since $C(u, v)=\iint \mathrm{d} x \mathrm{~d} y O(x, y) \exp (-2 \pi i(u x+v y))$. In the optical and infrared domain, only $V_{(u, v)}$, the ratio of coherent flux to the total flux can be safely measured, and even then its phase is not readily measurable by current

Further author information: (Send correspondence to G.D.)

G.D.: E-mail: Gilles.Duvert@univ-rhone-alpes.fr, Telephone: 133476514885 
Table 1. OIFITS version 1 tables and their main use.

\begin{tabular}{lll}
\hline HDU1 & Intent & Multiplicity \\
\hline (Header) & (Mostly empty) & \\
OI_TARGET & "Objects" positions (and effective cross-index) & 1 \\
OI_ARRAY & Geometry of telescope positions, aperture size & $0 . . \mathrm{N}$ \\
OI_WAVELENGTH & Observing wavelengths (and instrument identification tag) & $1 . . \mathrm{N}$ \\
OI_VIS2 & $V^{2}$ observables (baselines) & $0 . . \mathrm{N}$ \\
OI_T3 & phase closures (triplets of baselines) & $0 . . \mathrm{N}$ \\
OI_VIS & Differential Phases etc... & $0 . . \mathrm{N}$ \\
\hline
\end{tabular}

1: Header Data Unit, the highest level component of the FITS file structure (binary tables for OIFITS)

instruments. As described in Pauls et al. ? and Duvert et al. ?, many "proxies" for the real complex coherent flux are measurable and must be accounted for in the format: the modulus squared of the complex visibility $V_{(u, v)}^{2}=\left|V_{(u, v)}\right|^{2}$, the closure phase of a triplet of apertures $C P_{\left(u_{1}, v_{1}, u_{2}, v_{2}\right)}=\operatorname{Arg}\left[V_{\left(u_{1}, v_{1}\right)} V_{\left(u_{2}, v_{2}\right)} V_{\left(u_{1}+u_{2}, v_{1}+v_{2}\right)}^{*}\right]$, the differential visibility phase between two wavelengths $\lambda_{1}$ and $\lambda_{2}$, etc...

\section{FROM OIFITS1 TO OIFITS2}

The format was initially intended to support only the storage of calibrated, time-averaged data, following the example of the UV-FITS format ? in use in radioastronomy at the time. In an ideal world, calibrated observables produced by different interferometers have lost any instrument-specific effect or bias and can be merged, for example to be fitted together by one model, or to produce an image by inversion techniques. In the real world, systematics will be present, and it is necessary to add information about the beam recombiner, the interferometer array, etc, to the OIFITS format, similarly to the FITS-IDI format used in Radio Interferometry ${ }^{\text {. Nevertheless, }}$ by supporting only calibrated data - at least in the intent - the data description is simpler, closer to Physics, and also of small size, readily exchangeable and easily ingested by user programs.

Thus, the OIFITS format uses FITS?,? and provides separate tables for each "subsystem" and "kind of observable". Table ?? shows the FITS binary table names defined by version 1 of the norm and their intent.

\subsection{New Requirements for OIFITS2}

Following the establishment of a working group on interferometry data standards within IAU's Commission 54 , the requirements for evolution of OIFITS have been collected over a period of two years on the JMMC forum $^{\ddagger}$, with active participation of many community interferometrists and representatives of all past and future instruments and arrays. A draft was in circulation for one year, and has been deposited on ArXiv ${ }^{\S}$ where it can be "maintained" by the authors following returns from the community. It is intended that a refereed publication will be made at some point.

The changes were necessary due to multiple causes:

1. New Instrument needs, such as the polarised modes of VLTI/GRAVITY (see Paper 9907-33? ${ }^{\text {) }}$.

2. Data reduction needs (see Papers $9907-3^{?}$ and $9907-48^{?}$ ) requiring fluxes and/or spectra to reconstruct or fit the true brightness distribution of objects. Also, these programs need an adequate description of the statistical correlation between quantities.

3. DataBase requirements such as presented in Paper $9907-142^{\text {? }}$, needing numerous meta-data for bookkeeping and easy data-mining.

4. Experience from 10 years of OIFITS1 (exemplified by the various Beauty Contests, see Paper 9907-47?), showing unforeseen usage, such as storing "raw" or uncalibrated visibilities (VLTI/AMBER) and highlighting the need for more explicit rules.

5. "New" (or variants of "classical") Optical Interferometry observables, such as a ratio of squared visibilities?

\footnotetext{
$\ddagger_{\text {www }}$ jmmc.fr/twiki/bin/view/Jmmc/OIFITSTwoProject

${ }^{\S}$ http://arxiv.org/abs/1510.04556
} 